\title{
Human Metapneumovirus Impairs Apoptosis of Nasal Epithelial Cells in Asthma via HSP70
}

\author{
Engin Baturcam ${ }^{a, b}$ Natale Snape ${ }^{b}$ Tiong Han Yeo $^{e}$ Johanna Schagen ${ }^{b}$ \\ Emma Thomas $^{b}$ Jayden Logan ${ }^{b}$ Sally Galbraith ${ }^{b}$ Natasha Collinson ${ }^{c, d}$ \\ Simon Phipps f,g Emmanuelle Fantino $^{b}$ Peter D. Sly ${ }^{b}$ Kirsten M. Spannc, d \\ ${ }^{a}$ School of Medicine and ${ }^{b}$ Child Health Research Centre, The University of Queensland, ' $S c h o o l$ of Biomedical \\ Sciences, Queensland University of Technology, and ${ }^{\mathrm{d}}$ Institute for Health and Biomedical Innovation at the Centre \\ for Children's Health Research, Brisbane, Qld., Schools of ${ }^{\mathrm{e} C h e m i s t r y}$ and Molecular Biosciences and $\mathrm{f}^{\mathrm{B}}$ iomedical \\ Sciences, The University of Queensland, and ${ }^{9}$ Australian Infectious Disease Research Centre, St Lucia, Qld., Australia
}

\section{Key Words}

Asthma · Primary nasal epithelial cells $\cdot$ Human metapneumovirus · Apoptosis · Interferon · Heat shock protein 70

\begin{abstract}
Asthmatics are highly susceptible to respiratory viral infections, possibly due to impaired innate immunity. However, the exact mechanisms of susceptibility are likely to differ amongst viruses. Therefore, we infected primary nasal epithelial cells (NECs) from adults with mild-to-moderate asthma, with respiratory syncytial virus (RSV) or human metapneumovirus (hMPV) in vitro and investigated the antiviral response. NECs from these asthmatics supported elevated hMPV but not RSV infection, compared to non-asthmatic controls. This correlated with reduced apoptosis and reduced activation of caspase- 9 and caspase- $3 / 7$ in response to $\mathrm{hMPV}$, but not RSV. The expression of heat shock protein 70 (HSP70), a known inhibitor of caspase activation and subsequent apoptosis, was amplified in response to hMPV infection. Chemical inhibition of HSP70 function restored caspase
\end{abstract}

activation and reduced hMPV infection in NECs from asthmatic subjects. There was no impairment in the production of IFN by NECs from asthmatics in response to either hMPV or RSV, demonstrating that increased infection of asthmatic airway cells by hMPV is IFN-independent. This study demonstrates, for the first time, a mechanism for elevated hMPV infection in airway epithelial cells from adult asthmatics and identifies HSP70 as a potential target for antiviral and asthma therapies.

() 2016 S. Karger AG, Basel

\section{Introduction}

Human metapneumovirus (hMPV) and respiratory syncytial virus (RSV) cause acute respiratory tract disease in children and adults [1-3]. These viruses have been linked to the development of asthma in children [4] and are reported to exacerbate asthma in both children and adults $[5,6]$. Asthmatics are more susceptible to respiratory viral infections [7], reportedly due to deficient antiviral immunity. In cultured primary bronchial epithelial 
cells (BECs) from asthmatics, type I ( $\beta$ ) and type III $(\lambda)$ interferon (IFN) production is reduced in response to rhinovirus (RV) infection [8-10]. However, the association between asthma and reduced IFN production in response to other respiratory viruses remains unclear and controversial, particularly for those with mild-to-moderate asthma $[11,12]$.

Respiratory viruses first infect the airway epithelial cells (AECs) in the upper respiratory tract. However, less is known concerning the potential defects in antiviral defence associated with asthma in this compartment relative to the lower respiratory tract. We recently demonstrated that nasal epithelial cells (NECs) from atopic, wheezy children were IFN-competent in response to hMPV, although they produced significantly less IFN in response to RSV than NECs from healthy children. Regardless of differences in the IFN response, NECs from atopic, wheezy children supported elevated replication of both hMPV and RSV [13]. This suggests that IFN-independent mechanisms may contribute to elevated virus susceptibility of the asthmatic airway epithelium, and that virus-specific interactions influence the antiviral response of AECs. Due to the lack of understanding of antiviral responses in the upper airways and of AECs from adult asthmatics infected with viruses other than RV, we investigated the susceptibility of in vitro cultured primary NECs from adults with mild-to-moderate atopic asthma, to RSV and hMPV infection.

In addition to IFN-dependent antiviral responses, programmed cell death responses, such as apoptosis, are an integral part of innate immunity as they restrict viral replication and spread. Apoptosis occurs downstream of endoplasmic reticulum (ER) stress, which is triggered by viral protein synthesis and leads to the activation of the unfolded protein response (UPR) [14]. Sustained UPR signalling dysregulates the mitochondria and leads to the assembly of the apoptosome, which activates the intrinsic caspase cascade [15]. In this study, we found that NECs from atopic asthmatic adults were more susceptible to hMPV infection, but not RSV infection, than NECs from non-atopic non-asthmatics (or 'healthy controls'). We further demonstrate that this was independent of the IFN response. We therefore investigated cell death responses induced by ER stress and demonstrated that intrinsic apoptosis induced by active caspase- 9 and caspase- $3 / 7$ was reduced in association with asthma. This lack of caspase-activated apoptosis correlated with hMPV-induced expression of heat shock protein 70 (HSP70), which is known to dysregulate the function of the apoptosome [16]. Our study describes a mechanism for elevated sus- ceptibility of AECs from the upper respiratory tract of atopic asthmatic adults to hMPV infection, and identifies HSP70 as a potential therapeutic target to overcome this response to hMPV infection.

\section{Materials and Methods}

\section{Study Design, Subjects and Sample Collection}

Twenty community volunteers (10 atopic asthmatics and 10 non atopic, non-asthmatics) 20-47 years of age were recruited with $100 \%$ participation. The mean age was 26 years and $41 \%$ were male. Subjects with mild-to-moderate asthma were defined as those who had been diagnosed with asthma and used asthma medication on an as-needed basis. All of the subjects had well-controlled asthma, and none was using steroid medication at the time of enrollment. All asthmatic subjects reported aeroallergen sensitization (atopy). Active and former smokers were excluded from the study. None of the subjects was experiencing symptoms of respiratory tract infection at the time of study enrollment. Nasal epithelium scrapings were obtained from the inferior turbinate of each nare, using a purpose-designed curette as described previously [13]. Patients from the study population were chosen randomly where there were $<10$ samples in an experiment.

\section{Submerged Culture of Primary Epithelial Cells}

Sampled NECs were initially seeded into $25-\mathrm{cm}^{2}$ cell-culture flasks in steroid-supplemented bronchial epithelial growth medium (BEGM; Lonza). At passage 2, cells were seeded into 12- or 96well plates and cultured as submerged monolayers until $75 \%$ confluent [13]. The culture medium was then replaced with steroidfree medium $24 \mathrm{~h}$ prior to infection with RSV or hMPV.

\section{Infection with RSV or $h M P V$}

NECs were infected with either RSV (A2) or hMPV (CAN 9783 ) at a multiplicity of infection (MOI) of 3 plaque-forming units/ cell. Uninfected control cells were exposed to virus-free media. Virus stocks were prepared as previously described through sucrosecushion concentration followed by high-speed pelleting of viral particles to remove the sucrose [13]. Cells were incubated with virus for $2 \mathrm{~h}$ at $37^{\circ} \mathrm{C}$, and then washed 3 times with PBS, and then steroid-free culture medium (BEGM: Lonza) was added. Cells in 12 -well plates were lysed for total RNA extraction. Supernatants were stored for IFN secretion quantification. Cells in 96-well plates were used for live-cell caspase activation assays. Cells were sampled at indicated time points within $24 \mathrm{~h}$ post-infection (p.i.).

\section{Quantification of Viral RNA and Shed Virus}

Total RNA was extracted using RNAzol ${ }^{\circledR}$ (Molecular Research Center, Inc.) according to the manufacturer's instructions, and then reverse-transcribed using random hexamers (Applied Biosystems). RSV and hMPV intracellular RNA was quantified using SYBR green dye (QIAGEN), specific primers (online suppl. table S1; for all online suppl. material, see www. karger.com/doi/10.1159/000449101) and standard curves were generated using 10-fold serial dilutions of expression plasmids encoding the nucleocapsid (N) gene of RSV or hMPV (Biomatik). A plasmid encoding the $\beta$-actin gene (Biomatik) was used as a housekeeping standard. Viral gene copy number was calculated by plot- 
ting the cycle threshold on each standard curve. Random hexamers amplify both viral genomic RNA and mRNA, so total viral RNA was expressed in comparison to $\beta$-actin gene copy number. In order to quantify viral transcription in early infection, an oligo(dT) primer was used to reverse transcribe only mRNA (Life Technologies). HMPV $\mathrm{N}$ mRNA was then quantified in comparison to $\beta$-actin mRNA as above.

Infectious virus released into the supernatant was quantified using a standard immune-plaque assay in which rhesus monkey kidney epithelial cells (LLC-MK2 cells) were exposed to supernatants for $2 \mathrm{~h}$, then incubated under methyl cellulose for 7 days to allow plaque formation prior to fixation and immune-detection of hMPV-positive plaques [13].

IFN- $\beta$ and IFN- $\lambda_{1}$ Quantification

Cell supernatants were collected $24 \mathrm{~h}$ p.i. and secreted IFN quantified by ALPHAlisa (IFN- $\beta$; Perkin Elmer) and ELISA (IFN- $\lambda_{1}$; eBioscience) according to the manufacturer's instructions. Assay sensitivity was 9.6 and $8 \mathrm{pg} / \mathrm{ml}$, respectively.

\section{Annexin-V Staining for Apoptotic Cells}

NECs were detached from culture plates with Trypsin/EDTA 24 h p.i., stained with Annexin-V and 7-aminoactinomycin D (7AAD) (Enzo Life Sciences) and 20,000 events acquired using a LSR Fortessa flow cytometer (Becton Dickenson). Debris were gated out based on the cell size (FSC-A; forward scatter area) and granularity (SSC-A; side scatter area). 7-AAD (necrosis) and Annexin-V (apoptosis) plots were created and a quadrant was set for distinguishing the 4 populations: Q1-4: Annexin-V-/7-AAD+ (necrotic cells), Q2-4: Annexin-V+ /7-AAD+ (late apoptotic cells), Q3-4: Annexin-V- /7-AAD- (viable and non-apoptotic cells) and Q4-4: Annexin-V+ /7-AAD- (early apoptotic cells). Data was analyzed using FlowJo software (v10, Tree Star Inc.).

\section{Caspase Activity}

NECs were cultured in 96-well plates and the activation of caspase- $3 / 7$, caspase- 8 and caspase- 9 was quantified using luciferase expression assays according to the manufacturer's specifications (Promega) at time points between 2 and 24 h p.i. as indicated. For each time point, uninfected cells were used as assay controls and the results expressed as fold induction in infected cells compared to uninfected cells. In order to induce intrinsic apoptosis, cells at $75 \%$ confluence were treated with the $\mathrm{BH} 3$-only protein mimetic AT-101 (10 $\mu \mathrm{M}$; Sigma-Aldrich) or vehicle (BEGM + DMSO $0.035 \%)$. Cells were then sampled at 4,8 and $12 \mathrm{~h}$ post-treatment (p.t.).

\section{ER Stress and the UPR}

Total cellular RNA extracted as described above for viral RNA quantification was also used to quantify mRNA for markers of ER stress; Grp78 and sXBP1 by qRT-PCR at 8, 12 and 24 h p.i. (hMPV) and at $24 \mathrm{~h}$ p.i. alone (RSV). Downstream UPR markers CHOP, GADD34, BIM, PUMA and NOXA were quantified by qRT-PCR at 8,12 and 24 h p.i. with hMPV. Regulators of the apoptosome, Smac and XIAP, were quantified in the same way at $24 \mathrm{~h}$ p.i. with hMPV. Primers are listed in online supplementary table S1. Phosphorylation of the UPR marker c-Jun NH2-terminal kinase (JNK) was quantified at 12 and $24 \mathrm{~h}$ p.i. with hMPV by in-cell Western blotting of 96-well plate cultures using a commercial kit $\left(\mathrm{Face}^{\mathrm{TM}}\right.$ JNK, Active Motif).

\section{Western Blot}

HSP70 expression was quantified by Western blot analysis prior to infection and then at $16 \mathrm{~h}$ p.i. with hMPV. Whole-cell lysates were obtained using RIPA buffer supplemented with protein inhibitor cocktail (Thermo Scientific) and proteins were resolved using 4-12\% Bis-Tris gels (Life Technologies) according to the manufacturer's recommendations. Proteins were then transferred onto PVDF membranes $(0.45 \mu \mathrm{m}$, Millipore $)$ and probed with primary antibody against HSP70 (Enzo Lifesciences) and GAPDH (Cell Signaling). Secondary antibodies conjugated with IRDye $800 \mathrm{CW}$ (Rockland) were used and images were captured with the Odyssey Infrared Imaging System (LI-COR Biosciences). For densitometric quantification, the intensity of the bands was determined using Image Studio Lite software (LI-COR Biosciences).

\section{HSP70 Inhibition}

Cells were infected with hMPV as described above, then treated $8 \mathrm{~h}$ later with VER-155008 (50 $\mu \mathrm{M}$; Sigma-Aldrich) or vehicle (BEGM + DMSO 0.27\%), and then sampled at $16 \mathrm{~h}$ p.i. A noncytotoxic dose was empirically determined previously (not shown).

\section{Statistical Analysis}

Clustered and longitudinal data was analyzed using generalized estimating equations (GEE) [17]. Normal distribution was used as a model and an independent correlation structure as the working correlation matrix. The interactions between viral and host factors (clustered data) or time and viral factors (longitudinal data) were assessed with the Wald test (type III analysis). The Sidak test was used to adjust for multiple comparisons where a significant interaction was detected and $\mathrm{p}<0.05$ was considered statistically significant. All data analyses were conducted using IBM SPSS software v22 (Armonk, N.Y., USA).

\section{Results}

\section{Asthma Was Associated with Elevated hMPV \\ Infection Independent of the IFN Response}

HMPV infection, as quantified by total intracellular viral RNA, was 2.5 -fold $\left(0.4 \log _{10}\right)$ higher in NECs from asthmatic compared to non-asthmatic subjects at $24 \mathrm{~h}$ p.i. ( $p<0.05$; fig. 1a). RSV infection was not elevated compared to non-asthmatic subjects (fig. 1a).

Both hMPV and RSV significantly induced IFN- $\beta$ (fig. 1b) and IFN- $\lambda_{1}$ (fig. 1c) production by NECs (compared to uninfected NECs; $\mathrm{p}<0.0001$ ), with IFN- $\beta$ production being elevated in response to hMPV compared to RSV $(\mathrm{p}<0.01)$. However, IFN- $\beta$ and IFN- $\lambda_{1}$ production in response to either virus was not affected by asthma status (fig. $1 b, c$ ), and there was no relationship between IFN- $\beta$ or IFN- $\lambda_{1}$ production and viral RNA in NECs from asthmatic subjects (online suppl. fig. S1a, b). This demonstrates that NECs from asthmatic adults were IFN competent in response to RSV and hMPV, although this did not prevent elevated hMPV infection. 
Fig. 1. HMPV infection is elevated in NECs from asthmatics with no associated defect in IFN production. a RSV or hMPV N gene copy number was quantified by RT-qPCR using total RNA extracted from NECs $24 \mathrm{~h}$ p.i. (MOI 3) and control plasmids for RSV and hMPV N gene and $\beta$-actin gene expression. b Secreted IFN- $\beta$ was quantified by ALPHAlisa. c Secreted IFN- $\lambda_{1}$ was quantified by ELISA. $\mathrm{n}=10$ for both asthmatics and non-asthmatics. The bottom and top of the box plots represent the 5th and 95th percentiles, the bar represents the median and the whiskers represent minimum and maximum. A GEE approach was used to compare groups and the interaction between disease status and virus was significant for the viral load but not for the IFN response. ${ }^{*} \mathrm{p}<0.05,{ }^{* *} \mathrm{p}<0.01$ and $* * * * \mathrm{p}<0.0001$ show statistical significance.

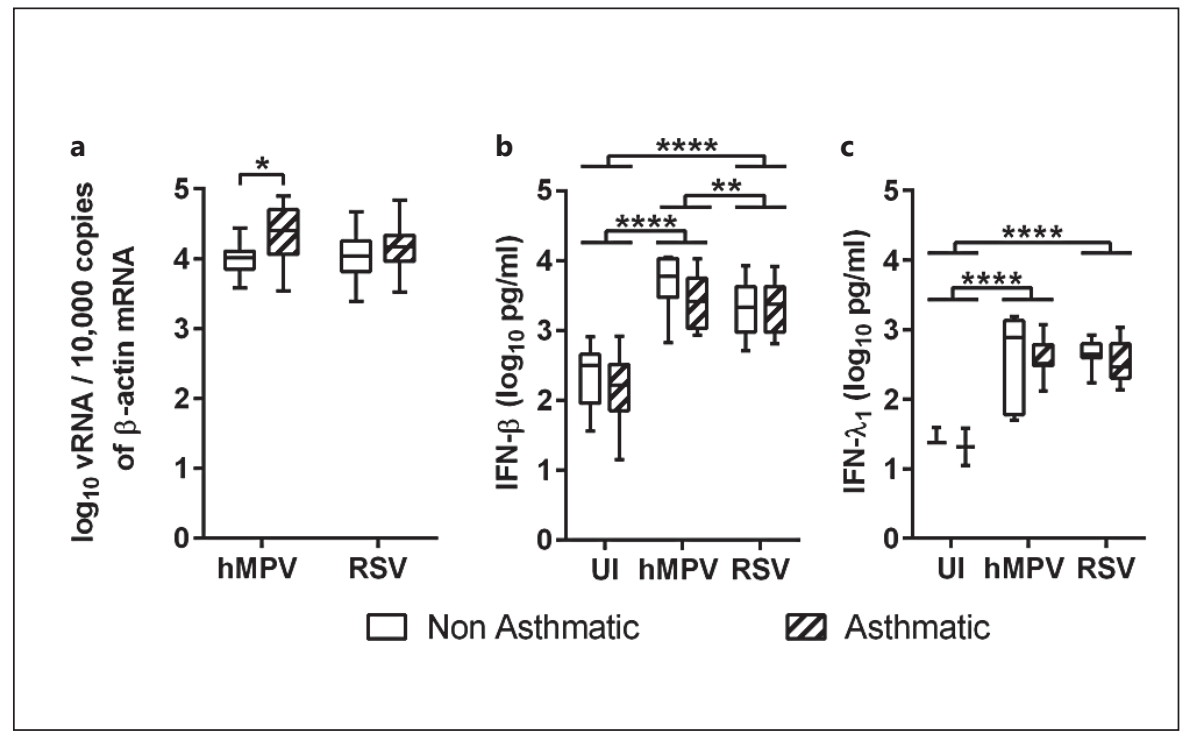

To address the possibility that viral attachment and entry by hMPV was elevated in NECs from asthmatics, hMPV N mRNA was quantified from 2 to $24 \mathrm{~h}$ p.i. as an indicator of early transcription events immediately after viral entry. Viral transcription was the same in NECs from asthmatic and non-asthmatic subjects for the first $12 \mathrm{~h}$ p.i., indicating no difference in the amount of viral genomic template available for transcription early in infection (online suppl. fig. S2). However, $\mathrm{N}$ mRNA was elevated in NECs from asthmatics compared to non-asthmatics at $24 \mathrm{~h}$ p.i. $(\mathrm{p}<0.05)$, as it was when total intracellular viral RNA was quantified (fig. 1a).

\section{Asthma Was Associated with Delayed and Reduced} Apoptosis in Response to hMPV

HMPV induced apoptosis, as quantified by flow cytometry of Annexin- $\mathrm{V}$-positive cells from both asthmatic and non-asthmatic subjects in comparison to uninfected cells at 24 h p.i. (2.8-fold, p $<0.0001$ and 1.5-fold, $\mathrm{p}<$ 0.0001 , respectively; fig. 2 ; online suppl. fig. S3b). Interestingly, the percentage of Annexin-V-positive uninfected NECs from asthmatics was significantly higher than from non-asthmatics. This may reflect a heightened sensitivity of asthmatic cells to the handling required for flow cytometry. Therefore, the percentage of hMPV-infected, Annexin-V-positive NECs was normalized against uninfected baseline expression for each subject group. Annexin- $\mathrm{V}$ detection was significantly lower for NECs from asthmatic subjects than from non-asthmatic subjects (1.9-fold, $\mathrm{p}<0.0001$; fig. 2b). This impaired apoptotic response to hMPV associated with asthma correlated with retention of the viable cells at $24 \mathrm{~h}$ p.i. (3.3-fold and 1.8fold loss of viable NECs from non-asthmatics and asthmatics, respectively; online suppl. fig. S3c). RSV also induced significant apoptosis in NECs from both asthmatic and non-asthmatic subjects compared to uninfected cells (online suppl. fig. S3b). However, there was no significant difference in response between the subject groups (fig. 2b).

To identify the mechanism of defective apoptosis of NECs from asthmatic subjects, activation of the final executors of apoptosis, caspase- 3 and caspase-7, was quantified together from 2 to $24 \mathrm{~h}$ p.i with either RSV or hMPV. Caspase-3/7 was not activated in uninfected NECs from either asthmatics or non-asthmatics for $24 \mathrm{~h}$ p.i. (online suppl. fig. S4a), therefore, the activity in hMPV-infected cells was expressed as fold induction compared to uninfected cells. HMPV significantly induced caspase-3/7 activity in NECs from non-asthmatic subjects at $12 \mathrm{~h}$ p.i. compared to uninfected NECs (3fold, $p<0.001$ ) and infected NECs from asthmatic subjects (2.8-fold, $\mathrm{p}<0.01$; fig. $3 \mathrm{a}$ ). Caspase- $3 / 7$ activity in NECs from non-asthmatics then increased steadily from 12 to $24 \mathrm{~h}$ p.i. ( 4.7 -fold at $16 \mathrm{~h}, \mathrm{p}<0.0001$ and 8 -fold at $24 \mathrm{~h}, \mathrm{p}<0.0001$, compared to uninfected NECs). However, caspase-3/7 activity in NECs from asthmatic subjects remained significantly lower than in NECs from non-asthmatic subjects at 16 and $24 \mathrm{~h}$ p.i. $(\mathrm{p}<0.0001$ and $\mathrm{p}<0.001$, respectively). Only at $24 \mathrm{~h}$ p.i. was caspase- $3 / 7$ activity significantly induced by hMPV in NECs from asthmatic subjects (1.5-fold compared to uninfected NECs, $\mathrm{p}<0.05$; fig. 3a). RSV did not activate caspase-3/7 in NECs from either asthmatic or non-asthmatic subjects 
a

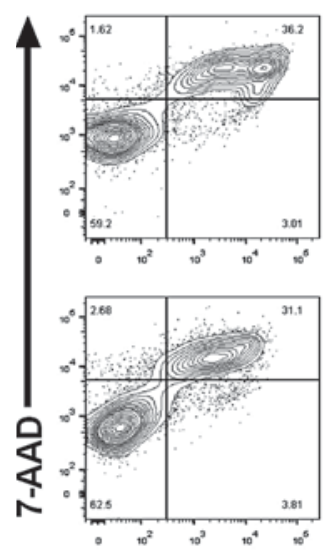

$+h M P V$
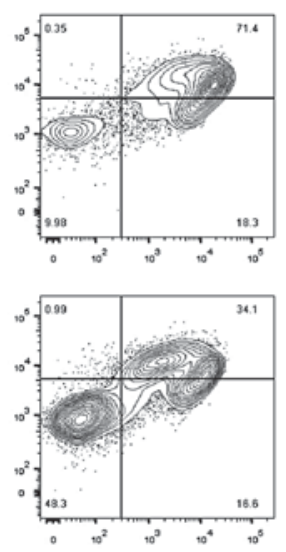

$+R S V$
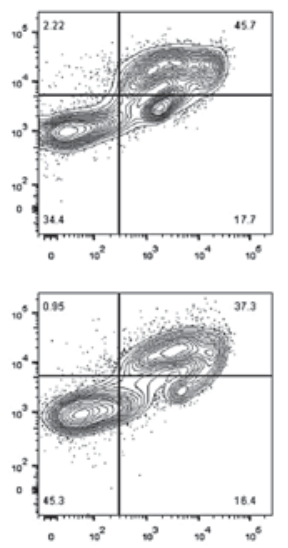

b

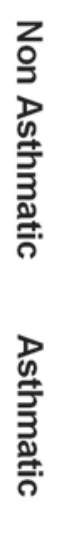

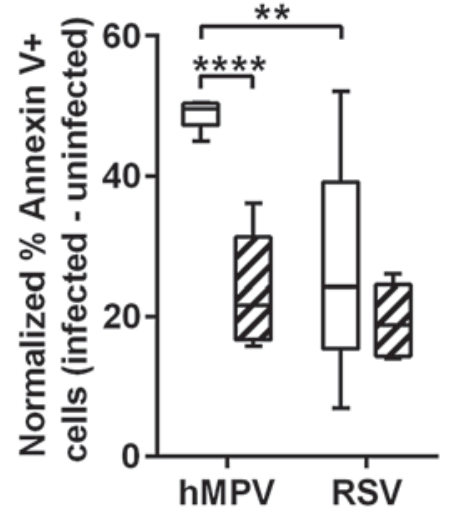

Non Asthmatic $\mathbb{Z}$ Asthmatic

Annexin V

Fig. 2. Apoptosis in response to hMPV is reduced in NECs from asthmatics. a Representative Annexin-V/7-AAD staining of NECs infected with hMPV or RSV at a MOI of 3 at 24 h p.i. b Quantification of the proportion of Annexin-V+ NECs at $24 \mathrm{~h}$ p.i. when normalized with Annexin-V-positive uninfected cells. $\mathrm{n}=5$ for both asthmatics and non-asthmatics. The bottom and top of the box plots represent the 5 th and 95 th percentiles, the bar represents the median and the whiskers represent minimum and maximum. A GEE approach was used to compare groups and the interaction between disease status and virus was significant. Statistical significance between asthmatic and non-asthmatic NECs is indicated by ** $\mathrm{p}<0.01$ and ${ }^{* * * *} \mathrm{p}<0.0001$. (fig. 3b), suggesting that RSV and hMPV induce cell death via different mechanisms.

The initiators, caspase- 9 and caspase- 8 lead to the downstream cleavage and activation of caspase-3/7. HMPV induced significantly elevated caspase- 9 activity in NECs from non-asthmatic subjects at $24 \mathrm{~h}$ p.i. (3.4-fold compared to uninfected NECs, $\mathrm{p}<0.01$; fig. 3c). Caspase- 9 activity was lower in asthmatic subjects than in non-asthmatic subjects at $24 \mathrm{~h}$ p.i., although this difference did not reach statistical significance (2.2-fold, $\mathrm{p}=$ $0.399)$. RSV did not induce caspase- 9 activation, again suggesting a different mechanism of apoptosis (fig. $3 \mathrm{~d}$ ). Caspase- 8 activation was poorly induced by hMPV in NECs from both asthmatic and non-asthmatic subjects (online suppl. fig. S4c). These data demonstrate that hMPV induced apoptosis in NECs via an intrinsic caspase- 9 and caspase-3/7 activation pathway, and that this response was impaired in NECs from asthmatic subjects. In addition to a disease-specific response, these data demonstrate a virus-specific response, as RSV induced significantly less apoptosis in non-asthmatic NECs and did not induce intrinsic caspase activation in NECs from either asthmatic or non-asthmatic subjects.
The Execution of Apoptosis in Asthmatic NECs Is Not Intrinsically Defective

To investigate whether defective caspase activation and apoptosis represented an inherent defect associated with asthma, NECs were treated with AT-101, which induces intrinsic apoptosis via the inhibition of Bcl2 proteins and up-regulation of the $\mathrm{BH} 3$-only proteins, PUMA and NOXA [18]. AT-101 significantly induced caspase-3/7 (fig. 3e) and caspase-9 (fig. 3f) activation within $8 \mathrm{~h}$ p.t., with sustained activation to $12 \mathrm{~h}$ p.t., in NECs from both asthmatic and non-asthmatic subjects. This demonstrates that there was no inherent defect in the ability of asthmatic NECs to undergo apoptosis. Rather, impaired caspase activation and apoptosis by NECs from asthmatics is an hMPV-specific mechanism.

\section{HMPV Induced ER Stress in NECs from Both \\ Asthmatic and Non-Asthmatic Subjects}

Intrinsic apoptosis is induced by sustained ER stress [15], which was identified in this study by elevated transcription of Grp78 (fig. 4a) and sXBP1 (fig. 4b) from 8 to 24 h p.i. in NECs from both asthmatic and non-asthmatic subjects. This suggests that hMPV did not suppress 


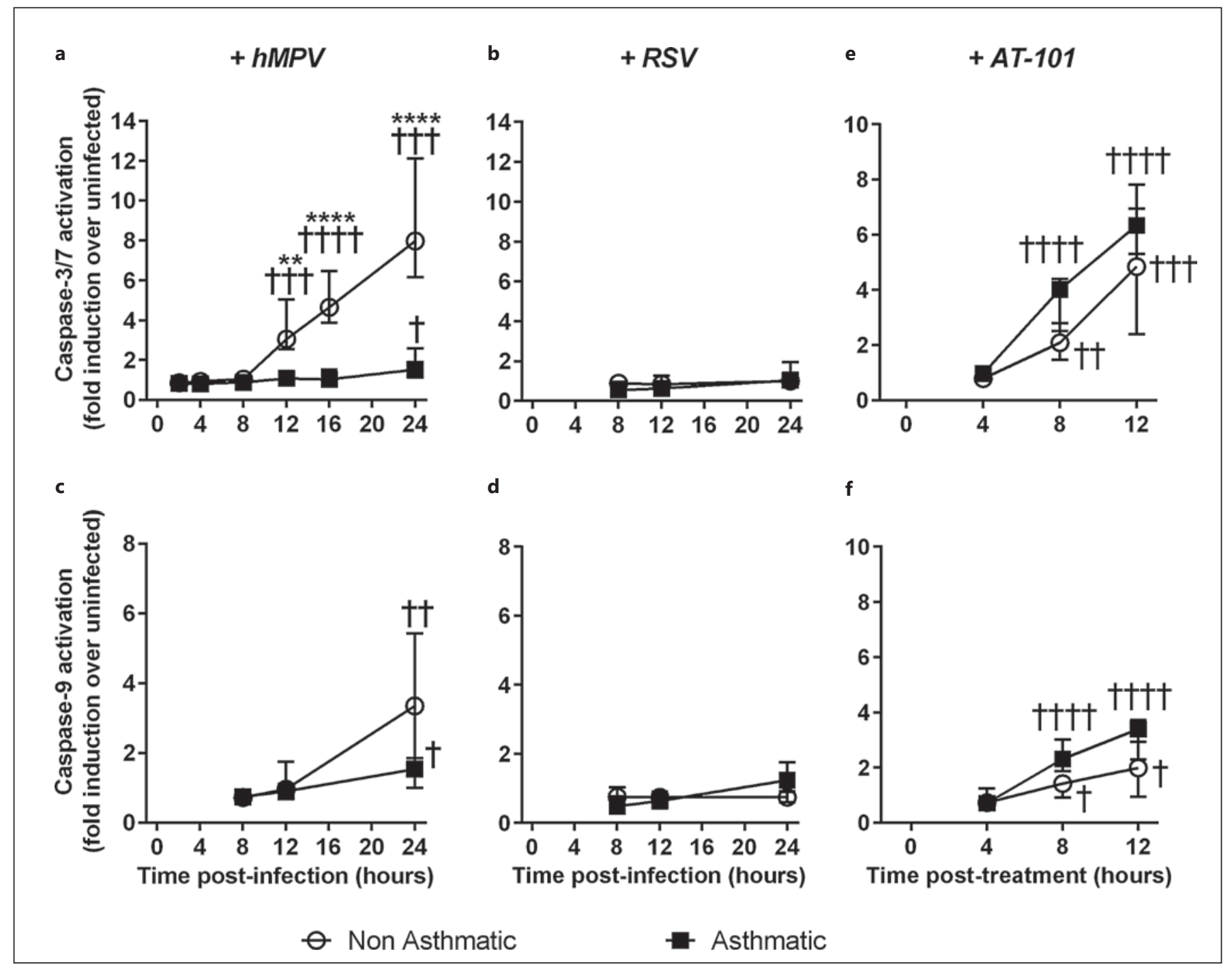

Fig. 3. Caspase- $3 / 7$ and caspase- 9 activity in NECs from asthmatic subjects is reduced in response to hMPV. Caspase-3/7 (a, b) and caspase-9 $(\mathbf{c}, \mathbf{d})$ activation was quantified by luminescence detection at the indicated time points within $24 \mathrm{~h}$ following hMPV or RSV infection (MOI 3). $\mathrm{n}=5$ for both asthmatics and non-asthmatics; median \pm range. Caspase- $3 / 7$ (e) and caspase- 9 (f) activation were quantified at 4,8 and $12 \mathrm{~h}$ after treatment with $10 \mu \mathrm{M}$ AT-101. $\mathrm{n}=5$ for both asthmatics and non-asthmatics; median \pm range. A GEE approach was used to compare groups and the interaction between disease status and time following hMPV infection was significant. Statistical significance between asthmatic and non-asthmatic NECs is indicated by ${ }^{* *} \mathrm{p}<0.01$ and ${ }^{* * * *} \mathrm{p}<$ 0.0001 . Statistical significance between infected (or treated) and uninfected (or untreated) NECs is indicated by ${ }^{\dagger} \mathrm{p}<0.05,{ }^{\dagger \dagger} \mathrm{p}<$ $0.01,{ }^{\dagger \dagger \dagger} \mathrm{p}<0.001$ and ${ }^{\dagger \dagger \dagger \dagger} \mathrm{p}<0.0001$. apoptosis in NECs from asthmatics via suppressed ER stress. As RSV did not induce caspase-3/7 or caspase-9 activity, we investigated if this correlated to poor upstream ER stress induction. RSV was a poor inducer of ER stress compared to hMPV, as both Grp78 (fig. 4a) and sXBP1 (fig. 4b) transcription were significantly lower at $24 \mathrm{~h}$ p.i. regardless of asthma status (non-asthmatic: 4.4fold, $\mathrm{p}<0.0001$ and asthmatic: 3.3 -fold, $\mathrm{p}<0.0001$; non- asthmatic: 2.8 -fold, $\mathrm{p}<0.0001$ and asthmatic: 3 -fold, $\mathrm{p}<$ 0.0001 , respectively).

\section{HMPV Induced a Maladaptive UPR in NECs from}

Both Asthmatic and Non-Asthmatic Subjects

Prolonged ER stress induces a maladaptive UPR through protein kinase RNA-like ER kinase (PERK) and inositol-requiring protein $1 \alpha$ (IREa) pathways [15]. 


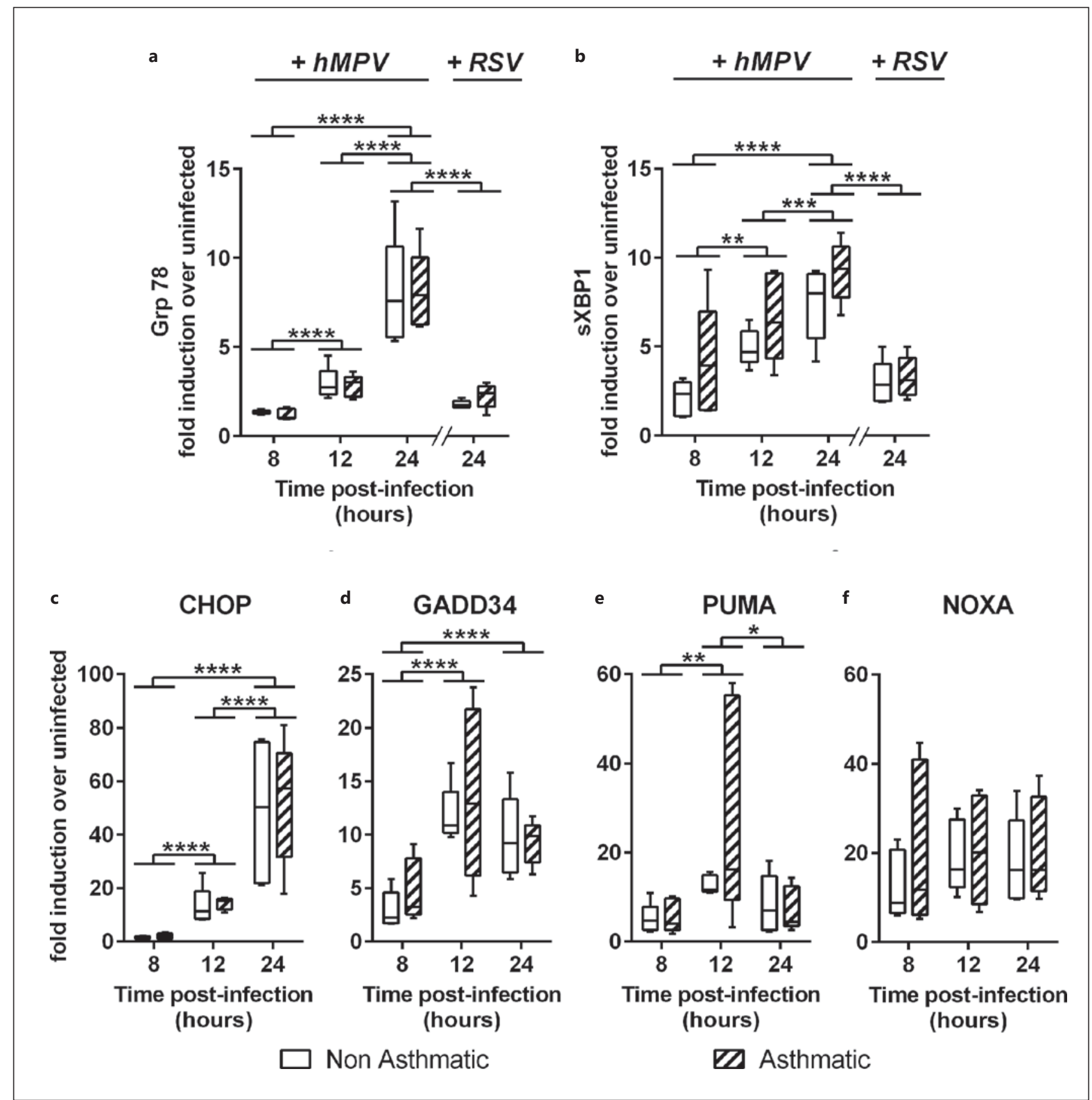

Fig. 4. HMPV induced the expression of ER stress and unfolded protein response markers in NECs from both asthmatic and nonasthmatic subjects. Grp78 (a), sXBP1 (b), CHOP (c), GADD34 (d), PUMA (e) and NOXA (f) mRNA expression in NECs at 8, 12 and $24 \mathrm{~h}$ following infection with hMPV or RSV (Grp78 and sXBP1 only; MOI of 3). RT-qPCR was performed using total cellular RNA. Fold induction was calculated using $2^{-\Delta \Delta C t}$ normalized to the expression of $\beta$-actin gene. $n=5$ for both asthmatic and non- asthmatic subjects. The bottom and top of the box plots represent the 5th and 95th percentiles, the bar represents the median and the whiskers represent minimum and maximum. A GEE approach was used to compare groups and the interaction between disease status and time (hMPV time course) or between disease status and virus (hMPV vs. RSV at $24 \mathrm{~h}$ p.i.) was not significant. ${ }^{*} \mathrm{p}<0.05$, ${ }^{* *} \mathrm{p}<0.01{ }^{* * *} \mathrm{p}<0.001$ and ${ }^{* * * *} \mathrm{p}<0.0001$ indicate statistical significance. 


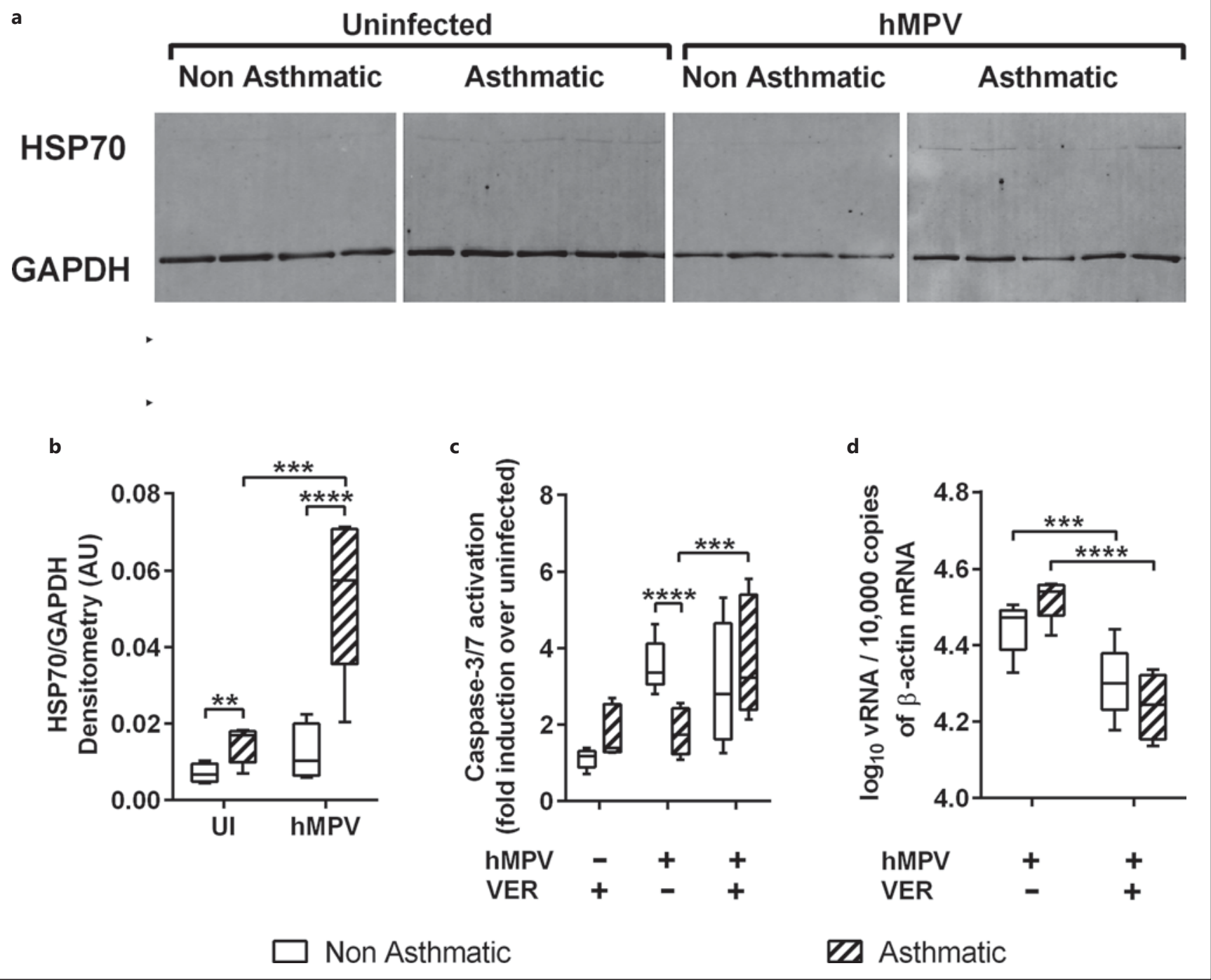

Fig. 5. HMPV induced elevated HSP70 expression by NECs from asthmatics that could be chemically inhibited to restore caspase activity and reduce the level of infection. a HSP70 and GAPDH expression analysis by Western blot at $16 \mathrm{~h}$ p.i. with hMPV (MOI of 3). The panel consists of representative immunoblots. b Signal intensity of HSP70 protein expression on Western blots was quantified by densitometry analysis normalized to GAPDH. The bottom and top of the box plots represent the 5 th and 95th percentiles, the bar represents the median and the whiskers represent minimum and maximum. $\mathrm{n}=4$ for non-asthmatic and 5 for asthmatic subjects. c, d NECs were infected with hMPV (MOI of 3 ) and then

HMPV did not activate the IRE1 $\alpha$ signalling pathway for UPR. This pathway induces apoptosis via phosphorylation of JNK, which then activates BIM and down-regulates anti-apoptotic Bcl-2. HMPV did not induce phosphorylation of JNK (online suppl. fig. S5) or induce the

HMPV Impairs Epithelial Cell Apoptosis in Asthma treated with VER-155008 (50 $\mu \mathrm{M}) 4$ h p.i. c Caspase-3/7 activity was determined by luminescence detection at $16 \mathrm{~h}$ p.i. d HMPV N gene copy number was quantified by RT-qPCR using total RNA extracted from NECs $16 \mathrm{~h}$ p.i. $\mathrm{n}=5$ for non-asthmatic and asthmatic subjects. The bottom and top of the box plots represent the 5th and 95th percentiles, the bar represents the median and the whiskers represent minimum and maximum. A GEE approach was used to compare groups and the interaction between disease status and hMPV (with or without VER treatment) was significant. ${ }^{* *} \mathrm{p}<0.01,{ }^{* * *} \mathrm{p}<0.001$ and ${ }^{* * * *} \mathrm{p}<0.0001$ show statistical significance.

expression of BIM (not shown). In NECs from both asthmatic and non-asthmatic subjects, hMPV induced the PERK pathway with elevated levels of the transcription factor CHOP in all NECs, from 8 to $24 \mathrm{~h}$ p.i. $(\mathrm{p}<0.0001$; fig. 4c). CHOP promotes sustained ER stress by increas- 
ing protein synthesis through GADD34 [15], which was also significantly induced in all NECs from 8 to $12 \mathrm{~h}$ p.i. $(\mathrm{p}<0.0001)$ and then remained steady up to $24 \mathrm{~h}$ p.i. (fig. 4d). CHOP also disrupts mitochondrial integrity by transcriptional up-regulation of the pro-apoptotic $\mathrm{BH} 3$ only proteins PUMA and NOXA [15]. PUMA was induced at $8 \mathrm{~h}$ p.i. and further induced by $12 \mathrm{~h}$ p.i but by 24 $\mathrm{h}$ p.i., induction had fallen (fig. 4e). NOXA was induced at $8 \mathrm{~h}$ p.i and did not significantly increase between 8 and $24 \mathrm{~h}$ p.i. (fig. 4f). Asthma was not associated with any modulation in the expression of these ER stress markers.

\section{HSP70 Expression Was Elevated in NECs from}

Asthmatics and Further Amplified by hMPV Infection

Intact ER stress signalling, but impaired caspase- 9 and caspase-3/7 activation suggested that the formation or function of the apoptosome was impaired in NECs from asthmatics. Apoptosome function is regulated by Smac/ Diablo inhibitor of apoptosis family proteins (XIAP) [19] and HSPs such as HSP70 [16]. Smac transcription was undetectable in NECs (not shown). XIAP transcription was induced by hMPV but was not differentially regulated in NECs from asthmatic subjects (online suppl. fig. S6). Conversely, we found that the expression of HSP70 protein in NECs from asthmatics was significantly elevated compared to in those from non-asthmatics, even prior to virus infection (2.6-fold, $\mathrm{p}<0.01$; fig. $5 \mathrm{a}, \mathrm{b}$ ). When infected with hMPV, HSP70 expression was further elevated in the NECs from asthmatics, but not in those from non-asthmatics, compared to uninfected NECs (4-fold, $\mathrm{p}<0.001)$. HSP70 transcription in response to hMPV was not detectable on $\mathrm{qPCR}$, despite utilising multiple primer sets (not shown). To assess the role of HSP70 in hMPVmediated inhibition of the apoptosome, hMPV-infected NECs were treated with VER-155008, which inhibits HSP70 activity by competitively binding to its ATPase domain and subsequently activating caspase-3/7 [16, 20]. Treatment with VER-155008 restored caspase-3/7 activity in the hMPV-infected NECs from asthmatics to a level similar to that of non-asthmatic NECs either infected with hMPV alone or infected and treated with VER155008 (fig. 5c). The inhibition of HSP70 activity reduced total intracellular viral RNA by approximately $50 \%$ in NECs from asthmatics $(\mathrm{p}<0.0001)$ and $30 \%$ in those from non-asthmatics ( $\mathrm{p}<0.001$; fig. $5 \mathrm{~d}$ ). Shed infectious virus was also reduced in VER-155008-treated NECs from asthmatics (online suppl. fig. S7). The reduction in viral RNA in non-asthmatic hMPV-infected NECs treated with VER-155008, with no corresponding increase in caspase-3/7 activation (fig. 5b), indicates that VER-
155008 also has an independent effect on hMPV replication. These data demonstrate that hMPV infection amplified an inherent overexpression of HSP70 in NECs from asthmatic adults, which then interfered with apoptosome formation to suppress the activation of caspase- 9 and caspase- $3 / 7$ that is required for the intrinsic apoptosis of infected cells. Impaired apoptosis then resulted in elevated hMPV infection in the NECs from asthmatic adults (fig. 6).

\section{Discussion}

In this study, we demonstrated a significant association between mild-to-moderate adult asthma and elevated hMPV infection in AECs from the upper respiratory tract. We further identified that elevated hMPV in association with asthma was due to a specific host-viral interaction in which hMPV induced HSP70 expression and inhibited the activation of caspases required for intrinsic apoptosis and viral clearance (fig. 6). This mechanism was independent of the IFN response, as NECs from both asthmatics and non-asthmatics produced IFN- $\beta$ and $-\lambda_{1}$ similarly, and the level of intracellular viral RNA was not correlated to the IFN response. An impaired IFN response by asthmatic individuals has been associated with increased viral susceptibility [8-10] and there is significant evidence that this impairment exists in haematopoietic cells, such as plasmacytoid dendritic cells [21]. However, our data adds to the accumulating evidence $[11,13$, 22] that AECs from mild-to-moderate asthmatic individuals are IFN-competent and that IFN-independent mechanisms contribute to elevated susceptibility to infection.

Programmed cell death pathways are activated by viral infection and play an important role in host defence by decreasing the number of infected cells and therefore limiting viral spread [23]. ER stress-induced apoptosis occurs via 2 main pathways: PERK and IRE1a. IRE1a signalling leads to the phosphorylation of JNK, which suppresses the anti-apoptotic function of Bcl-2 proteins [15]. PERK signalling leads to the up-regulation of pro-apoptotic CHOP/GADD153 transcription factors and the subsequent transcriptional activation of $\mathrm{BH} 3$-only proteins (BIM, PUMA and NOXA) [15], which alter mitochondrial integrity to initiate apoptosis through formation of the apoptosome. Here, we demonstrated that hMPV activated the PERK pathway of apoptosis via apoptosome formation in NECs, rather than the IRE1a pathway. The apoptosome is composed of cytochrome $c$ released by dysregulated mitochondria, apoptotic protease activating
60

J Innate Immun 2017;9:52-64 DOI: $10.1159 / 000449101$
Baturcam et al. 


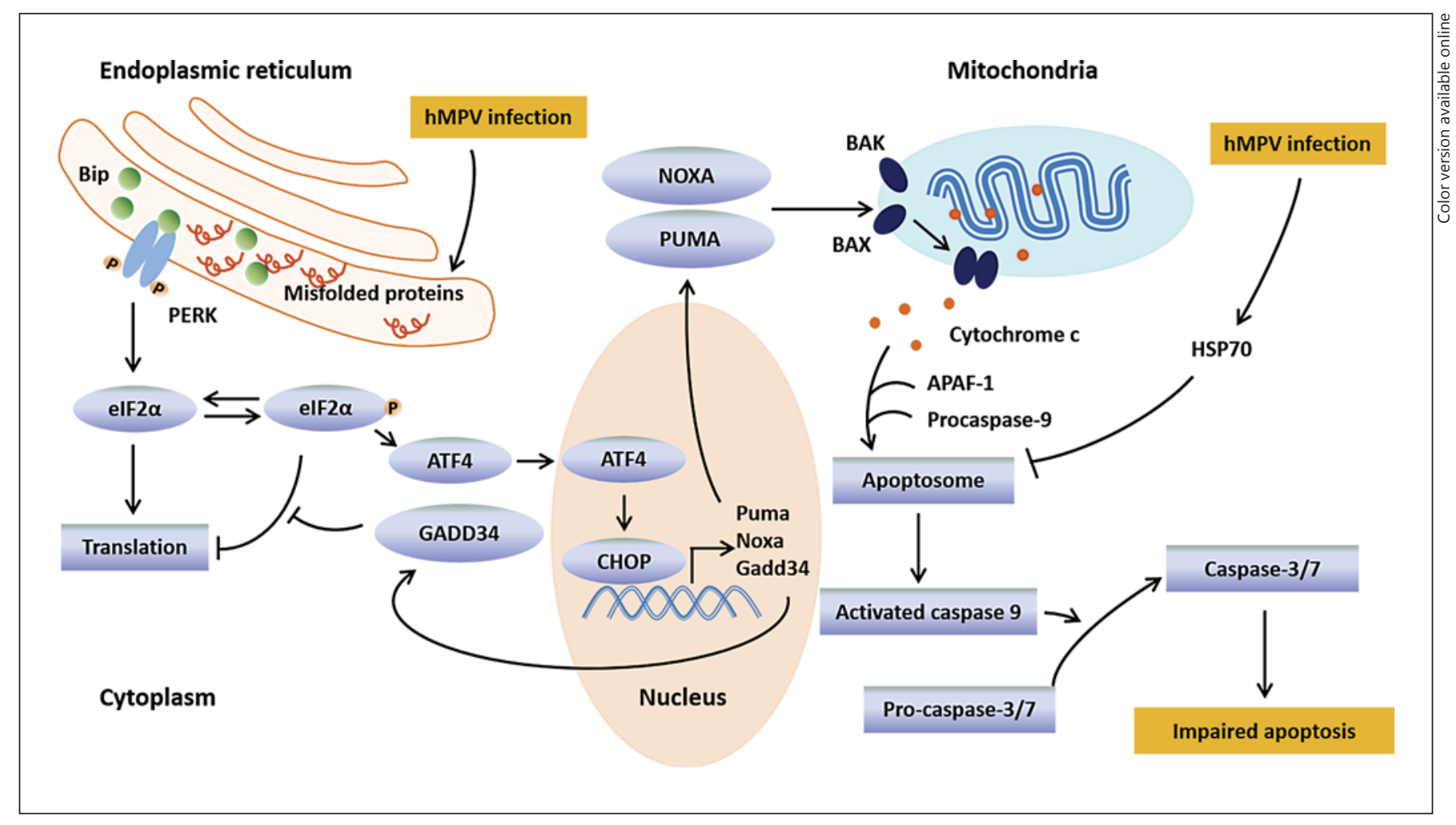

Fig. 6. Proposed model for impaired apoptotic response to hMPV infection in NECs from asthmatics. HMPV induced sustained ER stress and activated the maladaptive UPR through the PERK pathway. GADD34 maintained the ER burden and the pro-apoptotic $\mathrm{BH} 3$-only proteins NOXA and PUMA altered mitochondrial in- tegrity. However, downstream formation of the apoptosome was compromised by hMPV-induced HSP70, which impaired caspase- 9 and caspase-3/7 activation. This defect in the execution of intrinsic apoptosis resulted in elevated infection in NECs from asthmatic subjects. factor 1 (APAF-1) and pro-caspase-9. Its function is to cleave and activate caspase- 9 , which then initiates cleavage of the apoptosis effector caspase-3/7. The function of the apoptosome in NECs from asthmatics in this study was impaired in response to hMPV, such that the activation of caspase- 9 and caspase- $3 / 7$ was delayed and reduced. Interestingly, we observed caspase- $3 / 7$ activation $4 \mathrm{~h}$ earlier p.i. than caspase- 9 activation. This may be explained by the molecular timer function of the apoptosome. Pro-caspase- 9 causes the displacement of active caspase- 9 from the apoptosome complex, thereby limiting apoptosome activity and the accumulation of free, active caspase-9 [24]. In addition, the positive feedback loop of caspase- $3 / 7$ activation following its own cleavage may further contribute to this difference [25].

In this study, the lack of caspase- 8 signalling, which is mediated by cell surface death receptors, indicated that hMPV did not activate the extrinsic apoptosis pathway. Therefore, we investigated the mechanisms of intrinsic apoptosis that may be modulated by hMPV. ER stress and mitochondrial dysregulation induce intrinsic apoptosis in response to viral infection. However, the induction of key ER stress markers, maladaptive UPR markers and pro-apoptotic factors indicated that there was no hMPVinduced modulation of the PERK pathway that leads to apoptosome formation in association with asthma. We therefore investigated mechanisms by which hMPV may inhibit apoptosome function.

After demonstrating that the classic apoptosome modulators Smac and XIAP were not involved in apoptosome regulation in hMPV-infected NECs, we investigated HSP70 expression, as this is also known to inhibit apoptosome formation and subsequent intrinsic apoptosis to promote cell survival in lethal conditions [16]. We found that the NECs from asthmatic subjects inherently expressed more HSP70 protein than those from non-asthmatics. Interestingly, BECs from asthmatics have also been reported to express elevated baseline levels of HSP70 
$[26,27]$. HMPV infection induced further HSP70 expression in NECs from asthmatic subjects, suggesting a mechanism for impaired apoptosome function associated with hMPV infection in asthma. Importantly, we were able to inhibit the HSP70-binding function by treatment with VER-155008, which restored caspase-3/7 activation in NECs from asthmatics and suppressed hMPV infection. Although reduced hMPV infection following VER155008 treatment is likely the result of elevated apoptosis, a reduced binding efficiency of HSP70 may have a direct impact on hMPV replication. HSPs are known to interact directly with viruses; for example, HSP90 is an integral part of the RSV replication complex [28]. However, the role of HSP70 in hMPV replication, if any, is unknown.

This study also demonstrated the specificity of viralcellular interactions. Unlike hMPV, asthma was not associated with elevated susceptibility to RSV infection, also reported in other studies [22]. RSV induced limited caspase- 9 and caspase- $3 / 7$ activation in NECs regardless of asthma status but, according to Annexin- $V$ staining, it did induce cell death. This suggests that RSV employs a different mechanism of cell death and warrants further investigation. RSV can suppress apoptosis by the function of the 2 non-structural proteins NS1 and NS2 [29], and is known to induce the peroxidation of lipids in AECs through oxidative stress [30], which was recently associated with non-apoptotic cell death [31]. RSV was also a poor inducer of ER stress compared to hMPV, potentially due to the ability of RSV to prevent phosphorylation of eIF $2 \alpha$, and therefore the UPR, in order to ensure continuous global protein translation [32]. RSV is less prevalent in the exacerbation of adult asthma than of child asthma; this corresponds with our findings, in that RSV infection was not elevated in NECs from asthmatic subjects. RV, however, is a common cause of asthma exacerbation in adults, and RV-infected BECs from asthmatic subjects have demonstrated delayed and reduced caspase-3/7 activity and apoptosis [8]. These findings suggest that viruses interact differently with AECs from asthmatics to influence cell death responses and subsequent susceptibility to infection. Our study also revealed the absence of an inherent defect in the ability of NECs to undergo apoptosis when treated with a $\mathrm{BH} 3$ mimetic to induce caspase- 9 and caspase- $3 / 7$ activity, further supporting the hypothesis that impaired apoptosis of NECs in association with asthma is the result of specific viral mechanisms.

These data also suggest that asthmatic adults may be more susceptible to upper respiratory tract infection by hMPV than is currently considered to be the case. The true nature of the association between hMPV and asthma is not clear, as few epidemiological studies have been undertaken since hMPV was identified in 2001 [33].

A limitation of this study is the use of laboratory strains RSV-A2 and hMPV-CAN97-83. Other studies using primary adult BECs have demonstrated differences in cytopathogenicity and cytokine response amongst RSV strains [34]. Although similar studies using hMPV clinical isolates are lacking, the virus strain is an important determinant of the antiviral response and requires further investigation. Therefore, these data demonstrate a mechanism for elevated infection and reduced caspase driven intrinsic apoptosis in NECs from asthmatics in response to the CAN97-83 strain. Whether this is relevant for infection by other hMPV isolates will require further investigation.

Our in vitro modelling of infection of AECs was performed using submerged cultures and a MOI of 3 . This approach ensured synchronicity of initial infection and response within an infected cell population, thus maximizing the identification of differences based on disease status. However, a direct effect of high MOI is the rapid death of a population of cells within a few viral life cycles. This does not necessarily reflect a physiological response in which a virus will initially only infect some cells and then undergo several rounds of replication within a cell population. Also, submerged monolayers of cells are basal in morphology. Further investigation of cell death in response to RSV and hMPV infection in fully differentiated air-liquid interface cultures is worthwhile as this is a more physiologically relevant model.

Another factor that needs to be acknowledged in this study is the elevated Annexin-V staining amongst uninfected NECs from asthmatics. These same NEC cultures did not demonstrate intrinsically higher caspase-3/7 activity in the absence of hMPV. It is possible that apoptosis was induced in these cells by the manual handling required for flow cytometry, as apoptosis is more readily induced in asthmatic BECs than non-asthmatic BECs when exposed to non-viral stress [35].

Our data indicates that HSP70 may be a viable target to reduce hMPV infection in the asthmatic airway epithelium. HSPs are associated with tumour growth in cancer and elevated resistance to chemotherapy [36]. HSP70 is overexpressed in pancreatic cancer cells, confers resistance to apoptosis and is currently a target for a promising anti-cancer strategy [37]. HSP70 is also involved at multiple stages of viral life cycles [38], and disrupting this interaction by inhibiting HSP70 [39] or knocking it down [40] has already demonstrated promising antiviral outcomes. A therapeutic approach to asthma based on inhib-
62

J Innate Immun 2017;9:52-64 DOI: $10.1159 / 000449101$
Baturcam et al. 
iting the interaction between HSP70 and hMPV may be a potential strategy for decreasing the burden of hMPVinduced asthma exacerbations.

\section{Acknowledgements}

We thank Dr. Dimitrios Vagenas from the Institute for Health and Biomedical Innovation, Queensland University of Technology, for statistical advice. This study was supported by a National Health and Medical Research Council of Australia project grant (No. 1061921) awarded to K.M.S., E.F. and P.D.S. E.B. was supported by a Queensland Children's Medical Research Institute PhD support scholarship.

\section{Statement of Ethics}

This study was approved by the human ethics committees of the Lady Cilento Children's Hospital (LCCH), and the University of Queensland, Brisbane, and a written informed consent was obtained from every subject, thereby meeting the standards of the Declaration of Helsinki in its revised version of 1975 and its amendments of 1983, 1989 and 1996 [JAMA 1997;277:925-926].

\section{Disclosure Statement}

The authors declare no competing interests.

\section{References}

1 Hall CB, Weinberg GA, Iwane MK, Blumkin AK, Edwards KM, Staat MA, Auinger P, Griffin MR, Poehling KA, Erdman D, Grijalva CG, Zhu Y, Szilagyi P: The burden of respiratory syncytial virus infection in young children. N Engl J Med 2009;360:588-598.

2 Edwards KM, Zhu Y, Griffin MR, Weinberg GA, Hall CB, Szilagyi PG, Staat MA, Iwane M, Prill MM, Williams JV; New Vaccine Surveillance Network: Burden of human metapneumovirus infection in young children. N Engl J Med 2013;368:633-643.

3 Cesario TC: Viruses associated with pneumonia in adults. Clin Infect Dis 2012;55:107113.

4 Carroll KN, Wu P, Gebretsadik T, Griffin MR, Dupont WD, Mitchel EF, Hartert TV: The severity-dependent relationship of infant bronchiolitis on the risk and morbidity of early childhood asthma. J Allergy Clin Immunol 2009;123:1055-1061.

5 Carroll KN, Hartert TV: The impact of respiratory viral infection on wheezing illnesses and asthma exacerbations. Immunol Allergy Clin North Am 2008;28:539-561.

6 Westerly BD, Peebles RS Jr: Respiratory syncytial virus infections in the adult asthmatic - mechanisms of host susceptibility and viral subversion. Immunol Allergy Clin North Am 2010;30:523-539.

7 Busse WW, Lemanske RF Jr, Gern JE: Role of viral respiratory infections in asthma and asthma exacerbations. Lancet 2010;376:826834.

8 Wark PA, Johnston SL, Bucchieri F, Powell R, Puddicombe S, Laza-Stanca V, Holgate ST, Davies DE: Asthmatic bronchial epithelial cells have a deficient innate immune response to infection with rhinovirus. J Exp Med 2005; 201:937-947.

9 Contoli M, Message SD, Laza-Stanca V, Edwards MR, Wark PA, Bartlett NW, Kebadze T, Mallia P, Stanciu LA, Parker HL, Slater L, Lewis-Antes A, Kon OM, Holgate ST, Davies
DE, Kotenko SV, Papi A, Johnston SL: Role of deficient type III interferon-lambda production in asthma exacerbations. Nat Med 2006; 12:1023-1026.

10 Edwards MR, Regamey N, Vareille M, Kieninger E, Gupta A, Shoemark A, Saglani S, Sykes A, Macintyre J, Davies J, Bossley C, Bush A, Johnston SL: Impaired innate interferon induction in severe therapy resistant atopic asthmatic children. Mucosal Immunol 2013;6:797-806.

11 Sykes A, Macintyre J, Edwards MR, Del Rosario A, Haas J, Gielen V, Kon OM, McHale M, Johnston SL: Rhinovirus-induced interferon production is not deficient in well-controlled asthma. Thorax 2014;69:240-246.

12 Schwantes EA, Manthei DM, Denlinger LC, Evans MD, Gern JE, Jarjour NN, Mathur SK: Interferon gene expression in sputum cells correlates with the Asthma Index Score during virus-induced exacerbations. Clin Exp Allergy 2014;44:813-821.

13 Spann KM, Baturcam E, Schagen J, Jones C, Straub CP, Preston FM, Chen L, Phipps S, Sly $\mathrm{PD}$, Fantino E: Viral and host factors determine innate immune responses in airway epithelial cells from children with wheeze and atopy. Thorax 2014;69:918-925.

14 He B: Viruses, endoplasmic reticulum stress, and interferon responses. Cell Death Differ 2006; 13:393-403.

15 Hetz C: The unfolded protein response: controlling cell fate decisions under ER stress and beyond. Nat Rev Mol Cell Biol 2012;13:89102.

16 Lanneau D, Brunet M, Frisan E, Solary E, Fontenay M, Garrido C: Heat shock proteins: essential proteins for apoptosis regulation. J Cell Mol Med 2008;12:743-761.

17 Zeger SL, Liang KY: Longitudinal data analysis for discrete and continuous outcomes. Biometrics 1986;42:121-130.

18 Meng Y, Tang W, Dai Y, Wu X, Liu M, Ji Q, Ji M, Pienta K, Lawrence T, Xu L: Natural
BH3 mimetic (-)-gossypol chemosensitizes human prostate cancer via $\mathrm{Bcl}-\mathrm{xL}$ inhibition accompanied by increase of Puma and Noxa. Mol Cancer Ther 2008;7:2192-2202.

19 Shiozaki EN, Chai J, Rigotti DJ, Riedl SJ, Li P, Srinivasula SM, Alnemri ES, Fairman R, Shi Y: Mechanism of XIAP-mediated inhibition of caspase-9. Mol Cell 2003;11:519527.

20 Massey AJ, Williamson DS, Browne H, Murray JB, Dokurno P, Shaw T, Macias AT, Daniels Z, Geoffroy S, Dopson M, Lavan P, Matassova N, Francis GL, Graham CJ, Parsons R, Wang Y, Padfield A, Comer M, Drysdale MJ, Wood M: A novel, small molecule inhibitor of $\mathrm{Hsc70/Hsp70} \mathrm{potentiates} \mathrm{Hsp90} \mathrm{inhibitor} \mathrm{in-}$ duced apoptosis in HCT116 colon carcinoma cells. Cancer Chemother Pharmacol 2010;66: 535-545.

21 Lynch JP, Mazzone SB, Rogers MJ, Arikkatt JJ, Loh Z, Pritchard AL, Upham JW, Phipps S: The plasmacytoid dendritic cell: at the crossroads in asthma. Eur Respir J 2014;43:264275.

22 Patel DA, You Y, Huang G, Byers DE, Kim HJ, Agapov E, Moore ML, Stokes Peebles R, Jr, Castro M, Sumino K, Shifren A, Brody SL, Holtzman MJ: Interferon response and respiratory virus control are preserved in bronchial epithelial cells in asthma. J Allergy Clin Immunol 2014;134:1402-1412.

23 Mahalingam S, Meanger J, Foster PS, Lidbury BA: The viral manipulation of the host cellular and immune environments to enhance propagation and survival: a focus on RNA viruses. J Leukoc Biol 2002;72:429-439.

24 Malladi S, Challa-Malladi M, Fearnhead HO, Bratton SB: The Apaf- 1 *procaspase- 9 apoptosome complex functions as a proteolyticbased molecular timer. EMBO J 2009;28: 1916-1925.

25 McIlwain DR, Berger T, Mak TW: Caspase functions in cell death and disease. Cold Spring Harb Perspect Biol 2013;5:a008656. 
26 Vignola AM, Chanez P, Polla BS, Vic P, Godard P, Bousquet J: Increased expression of heat shock protein 70 on airway cells in asthma and chronic bronchitis. Am J Respir Cell Mol Biol 1995;13:683-691.

27 Bertorelli G, Bocchino V, Zhuo X, Chetta A, Del Donno M, Foresi A, Testi R, Olivieri D: Heat shock protein 70 upregulation is related to HLA-DR expression in bronchial asthma. Effects of inhaled glucocorticoids. Clin Exp Allergy 1998;28:551-560.

28 Munday DC, Wu W, Smith N, Fix J, Noton SL, Galloux M, Touzelet O, Armstrong SD, Dawson JM, Aljabr W, Easton AJ, RameixWelti MA, de Oliveira AP, Simabuco FM, Ventura AM, Hughes DJ, Barr JN, Fearns R, Digard P, Eleouet JF, Hiscox JA: Interactome analysis of the human respiratory syncytial virus RNA polymerase complex identifies protein chaperones as important cofactors that promote L-protein stability and RNA synthesis. J Virol 2015;89:917-930.

29 Bitko V, Shulyayeva O, Mazumder B, Musiyenko A, Ramaswamy M, Look DC, Barik S: Nonstructural proteins of respiratory syncytial virus suppress premature apoptosis by an NF-kappaB-dependent, interferon-independent mechanism and facilitate virus growth. J Virol 2007;81:1786-1795.
30 Hosakote YM, Liu T, Castro SM, Garofalo RP, Casola A: Respiratory syncytial virus induces oxidative stress by modulating antioxidant enzymes. Am J Respir Cell Mol Biol 2009;41: 348-357.

31 Dixon SJ, Lemberg KM, Lamprecht MR, Skouta R, Zaitsev EM, Gleason CE, Patel DN, Bauer AJ, Cantley AM, Yang WS, Morrison B 3rd, Stockwell BR: Ferroptosis: an iron-dependent form of nonapoptotic cell death. Cell 2012;149:1060-1072.

32 Groskreutz DJ, Babor EC, Monick MM, Varga SM, Hunninghake GW: Respiratory syncytial virus limits alpha subunit of eukaryotic translation initiation factor 2 (eIF2 $\alpha$ ) phosphorylation to maintain translation and viral replication. J Biol Chem 2010;285:2402324031.

33 van den Hoogen BG, de Jong JC, Groen J, Kuiken T, de Groot R, Fouchier RA, Osterhaus $\mathrm{AD}$ : A newly discovered human pneumovirus isolated from young children with respiratory tract disease. Nat Med 2001;7:719-724.

34 Villenave R, O'Donoghue D, Thavagnanam S, Touzelet O, Skibinski G, Heaney LG, McKaigue JP, Coyle PV, Shields MD, Power UF: Differential cytopathogenesis of respiratory syncytial virus prototypic and clinical isolates in primary pediatric bronchial epithelial cells. Virol J 2011;8:43.

35 Bucchieri F, Puddicombe SM, Lordan JL, Richter A, Buchanan D, Wilson SJ, Ward J, Zummo G, Howarth PH, Djukanovic R, Holgate ST, Davies DE: Asthmatic bronchial epithelium is more susceptible to oxidant-induced apoptosis. Am J Respir Cell Mol Biol 2002;27:179-185.
36 Ciocca DR, Calderwood SK: Heat shock proteins in cancer: diagnostic, prognostic, predictive, and treatment implications. Cell Stress Chaper 2005;10:86-103.

37 Chugh R, Sangwan V, Patil SP, Dudeja V, Dawra RK, Banerjee S, Schumacher RJ, Blazar BR, Georg GI, Vickers SM, Saluja AK: A preclinical evaluation of Minnelide as a therapeutic agent against pancreatic cancer. Sci Transl Med 2012;4:156ra139.

38 Nagy PD, Wang RY, Pogany J, Hafren A, Makinen K: Emerging picture of host chaperone and cyclophilin roles in RNA virus replication. Virology 2011;411:374-382.

39 Gonzalez O, Fontanes V, Raychaudhuri S, Loo R, Loo J, Arumugaswami V, Sun R, Dasgupta A, French SW: The heat shock protein inhibitor Quercetin attenuates hepatitis C virus production. Hepatology 2009;50:17561764.

40 Manzoor R, Kuroda K, Yoshida R, Tsuda Y, Fujikura D, Miyamoto H, Kajihara M, Kida $\mathrm{H}$, Takada A: Heat shock protein 70 modulates influenza A virus polymerase activity. J Biol Chem 2014;289:7599-7614. 